\title{
Estudio diagnóstico sobre la gestión de empresas saludables en la era del covid-19
}

\section{Diagnostic study on the management of healthy companies in the era of covid-19. Tirso Javier Hernández-Gracia ${ }^{a}$, Danae Duana-Avila ${ }^{b}$ Alejandra Corichi García ${ }^{c}$ Miriam López Vargas ${ }^{d}$}

\begin{abstract}
:
The objective of the research was to carry out a diagnostic study on the management of healthy companies in the era of Covid-19. The IBERMUTUA instrument was used with 100 workers from two educational institutions. The methodology follows a quantitative, descriptive and transversal approach. The main results show that, in general, organizations have a level of $44 \%$ in various aspects of health management.
\end{abstract}

Keywords:

Management, Health, Wellbeing, Companies, Covid-19

\section{Resumen:}

El objetivo de la investigación fue realizar un estudio diagnóstico sobre la gestión de empresas saludables en la era del Covid-19. Se utilizó el instrumento de IBERMUTUA con 100 trabajadores de dos instituciones educativas. La metodología sigue un enfoque cuantitativo, descriptivo y transversal. Los principales resultados muestran que en general las organizaciones poseen un nivel del $44 \%$ en diversos aspectos de gestión de salud.

Palabras Clave:

Gestión, Salud, Bienestar, Empresas, Covid-19

\section{Introducción}

Desde 1948 el concepto de salud fue definido por la Organización Mundial de la Salud (OMS) como un estado integral de bienestar físico, mental y social, es decir, no solo está relacionado con la ausencia de enfermedades patológicas, sino con aquella sensación de equilibrio que un ser humano puede tener al estar en contacto con distintos aspectos que son parte de su vida cotidiana. $\mathrm{Si}$ trasladamos el término personal al organizacional encontramos que ésta última se encuentra asociada a la efectividad de la empresa para reaccionar ante circunstancias comerciales cambiantes impuestas por el entorno y la competencia, siendo más eficientes y eficaces, evitando la rotación de trabajadores y manteniendo un desempeño significativo [1].

La salud organizacional y personal son términos que están asociados a la seguridad y salud ocupacional, donde las empresas realizan esfuerzos para prevenir accidentes laborales y enfermedades profesionales, diseñando para ello ambientes de trabajo cómodos y libres de peligros, persiguiendo principalmente tres

a Universidad Autónoma del Estado de Hidalgo. Programa educativo de Administración, https://orcid.org/0000-0003-0425-0800, Email: thernan@uaeh.edu.mx.

b Autor de Correspondencia, Universidad Autónoma del Estado de Hidalgo. Programa educativo de Administración. https://orcid.org/00000003-2286-2843, Email: duana@uaeh.edu.mx.

c Universidad Autónoma del Estado de Hidalgo. Programa educativo de Administración. https://orcid.org/0000-0002-4785-7234, Email: corichi@uaeh.edu.mx.

d Universidad Autónoma del Estado de Hidalgo, Alumna del 9no semestre de la Licenciatura en Administración del ICEA, https://orcid.org/0000-0003-3327-5388, Email: lo295574@uaeh.edu.mx. 
objetivos importantes: 1) Proteger a los empleados, las organizaciones y la producción de los bienes y/o servicios que ofrece; 2) Crear y fomentar ambientes de trabajo agradables; y 3) Garantizar un buen clima laboral y disminuir los riesgos [2].

A partir del 2020 las organizaciones no solo tienen que luchar con las variantes impuestas por el entorno económico, social y político del lugar donde se encuentran ubicadas, así como con las presiones del sector empresarial al que pertenecen, sino ahora sumar una variable que no estaba prevista "el virus covid-19", el cual llegó como un meteorito e impacto de manera descomunal sobre cada componente organizacional, pero sobre todo, desestabilizando y cambiando de manera repentina la salud mental de los trabajadores, generando angustia y depresión [3].

En marzo de 2020 la OMS declaró la pandemia originada por el coronavirus (Covid-19) a nivel mundial, con lo cual comienzan a presentarse cientos de casos de personas con problemas de enfermedades respiratorias, incluyendo fiebre tos seca, cansancio, dolores, entre otros síntomas [4].

Durante el año 2020 la incertidumbre por la magnitud de este evento y el miedo al no tener una vacuna disponible para enfrentar al virus fueron las principales preocupaciones a nivel mundial, se tomaron medidas drásticas de confinamiento entre la población y las empresas lo que causó una crisis económica mundial. Por supuesto esto fue motivo de miedo para los trabajadores de todos los sectores, especialmente los del sector salud [5].

Dimensionar los daños causados por esta pandemia es difícil, ya que se le considera la mayor crisis social y económica en décadas, aunque con el paso de los meses se ha podido calcular el impacto a corto y mediano plazo, como que la desigualdad de género aumentará, la pobreza se acrecentará, esto sin mencionar la crisis sanitaria. Regresar a la normalidad será todo un reto y algunas medidas de higiene seguramente perdurarán, es completamente necesaria la intervención del gobierno y la cooperación de la población [6].

Es sumamente importante ser una empresa con resiliencia para afrontar esta situación ya que de lo contrario corren el riesgo de desaparecer. Es necesario adaptarse a los cambios y aprender del trauma y volverse una organización más fortalecida ya que son las que triunfarán en esta crisis, las empresas saludables deben promover prácticas como el manejo del estrés, buenas relaciones entre colaboradores, trabajo en equipo y prevención de crisis, es importante saber manejar una crisis antes de que esta ocurra [7].

La salud organizacional y personal son dos términos que van de la mano, ya que el primero se convierte en el promotor de acciones encaminadas a mantener el cuidado de sus trabajadores, y el segundo, se encarga de seguir medidas que permitan protegerse a sí mismos y ser parte del bienestar de los demás.

Una buena parte de la vida del ser humano, principalmente cuando alcanza la mayoría de edad, se la pasa en su lugar de trabajo y es ahí precisamente el escenario idóneo para promover la salud organizacional y personal de los trabajadores, ya que la empresa posee las estructuras y los medios que pueden utilizarse para tal efecto, así como el interés conjunto de aspirar a obtener mejores niveles de eficacia, productividad y competitividad.

Llevar a cabo acciones de gestión en salud es responsabilidad de las empresas, teniendo en cuenta que para ello es necesario establecer acciones que permitan disminuir aquellos aspectos asociados con factores de riesgo, así como promover estrategias para el desarrollo del bienestar físico, mental y social de sus trabajadores.

\section{Conceptos de salud organizacional y personal}

Según la definición de la OMS, "la salud es un estado de completo bienestar físico, mental y social" [8]. La salud, por tanto, tiene varias dimensiones. Sin embargo, la mayoría de las veces, cuando pensamos en salud, pensamos en términos de salud física, porque es lo más tangible y lo más fácil de entender. Sin embargo, en la vida laboral y la economía modernas, la salud mental y social son cada vez más importantes.

En el cuadro 1, las tres dimensiones de la salud y la salud en su conjunto están relacionadas con la productividad y los beneficios económicos. Los tres aspectos de la salud están relacionados con el poder de producción, aspectos típicos del trabajo donde se usan estos poderes, tipos típicos de trabajo asociados con el uso de estos poderes, conceptos asociados de funciones del personal, herramientas típicas para usar estos recursos (en algunas circunstancias, las máquinas compiten con los seres humanos como recurso de producción (es notable que las mismas máquinas puedan funcionar como competidores o como facilitadores de la mano de obra), y el tipo de economía donde el uso de estos recursos es típico.

\begin{tabular}{|c|c|c|c|c|}
\hline $\begin{array}{c}\text { La salud } \\
\text { como } \\
\text { recurso }\end{array}$ & Salud física & Salud mental & Salud social & Salud total \\
\hline Potencia & $\begin{array}{c}\text { Potencia } \\
\text { muscular }\end{array}$ & $\begin{array}{c}\text { Potencia } \\
\text { cerebral }\end{array}$ & $\begin{array}{c}\text { Potencia } \\
\text { social y } \\
\text { comunicativa }\end{array}$ & $\begin{array}{c}\text { Potencia } \\
\text { humana / } \\
\text { hombre }\end{array}$ \\
\hline
\end{tabular}




\begin{tabular}{|c|c|c|c|c|}
\hline $\begin{array}{c}\text { Trabajo } \\
\text { típico }\end{array}$ & $\begin{array}{c}\text { Manejo de } \\
\text { materiales }\end{array}$ & $\begin{array}{c}\text { Procesamient } \\
\text { o de } \\
\text { información }\end{array}$ & $\begin{array}{c}\text { Trabajo en } \\
\text { red, gestión }\end{array}$ & $\begin{array}{c}\text { Creación y } \\
\text { desarrollo }\end{array}$ \\
\hline $\begin{array}{c}\text { Teoría de los } \\
\text { recursos }\end{array}$ & $\begin{array}{c}\text { Recursos } \\
\text { humanos } \\
\text { clásicos }\end{array}$ & $\begin{array}{c}\text { Capital } \\
\text { intelectual }\end{array}$ & Capital social & $\begin{array}{c}\text { Capital } \\
\text { humano y } \\
\text { social }\end{array}$ \\
\hline $\begin{array}{c}\text { Tecnologías } \\
\text { típicas: } \\
\text { competidora } \\
\text { s y /o } \\
\text { habilitadora } \\
\mathbf{s}\end{array}$ & $\begin{array}{c}\text { Tecnología } \\
\mathbf{s} \\
\text { tradicionale } \\
\mathrm{s}\end{array}$ & $\begin{array}{c}\text { Tecnología de } \\
\text { la información }\end{array}$ & $\begin{array}{c}\text { Tecnología de } \\
\text { la } \\
\text { comunicación } \\
\text { y el transporte }\end{array}$ & $\begin{array}{c}\text { Tanto la } \\
\text { tecnología } \\
\text { clásica como } \\
\text { la tecnología } \\
\text { de la } \\
\text { información y } \\
\text { la } \\
\text { comunicación } \\
\text { (TIC) }\end{array}$ \\
\hline $\begin{array}{c}\text { Herramienta } \\
\text { s típicas }\end{array}$ & $\begin{array}{c}\text { Herramient } \\
\text { as } \\
\text { mecánicas }\end{array}$ & $\begin{array}{c}\text { Computadora } \\
\mathrm{s}\end{array}$ & $\begin{array}{c}\text { Internet y } \\
\text { teléfonos } \\
\text { celulares, } \\
\text { automóviles y } \\
\text { aviones }\end{array}$ & $\begin{array}{c}\text { Herramientas } \\
\text { innovadoras e } \\
\text { integradas }\end{array}$ \\
\hline $\begin{array}{c}\text { Sociedad } \\
\text { económica }\end{array}$ & $\begin{array}{c}\text { Sociedad } \\
\text { industrial }\end{array}$ & $\begin{array}{c}\text { Economía del } \\
\text { conocimiento }\end{array}$ & $\begin{array}{c}\text { Sociedad en } \\
\text { red }\end{array}$ & $\begin{array}{c}\text { Economía } \\
\text { sostenible }\end{array}$ \\
\hline
\end{tabular}

Cuadro 1. Aspectos de salud y sus relaciones con la productividad y los beneficios económicos.

\section{Fuente Elaboración propia a partir de [9]}

Por supuesto, estas categorías no son absolutas. Los grupos enumerados en esta tabla se superponen, por ejemplo, el trabajo informático en el que se combinan la carga física estática y la carga mental. A menudo, esta combinación de exposiciones es el factor de riesgo real. A pesar de esto, la tabla sugiere que en la economía del conocimiento, la salud mental es el recurso dominante, mientras que en la sociedad en red, los aspectos sociales de la salud son cruciales. De hecho, para una economía sostenible, el uso de todo el potencial de la salud es cada vez más importante, lo que implica que tanto la salud mental como la social son cada vez más importantes [9]

\section{Antecedentes}

Los hechos que ponen en riesgo la vida o la salud del hombre han existido desde siempre. En consecuencia, también desde siempre, el hombre ha tenido la necesidad. de protegerse. Pero cuando estos hechos 0 condiciones de riesgo se circunscriben al trabajo, históricamente, el tema de la producción ha recibido mayor importancia que el de la seguridad, ya que es sólo recientemente que el hombre, como persona natural y como persona jurídica, ha tomado conciencia de la importancia que reviste la salud ocupacional y la seguridad en el trabajo. Ello no significa que no haya habido dignos antecedentes que preludiaron y que sirvieron de base para el actual campo de la seguridad. En ese sentido, el presente trabajo, pretende rescatar los eventos que constituyen hitos importantes para la institucionalización de la seguridad industrial como profesión y como disciplina científica dentro del marco de la historia.
Ahora bien, la salud ocupacional y la seguridad industrial conforman un binomio inseparable que garantiza la minimización de los riesgos laborales y la prevención de accidentes en el trabajo 1. Por riesgo laboral se entiende la probabilidad de que ocurran lesiones a las personas, daños al medio ambiente 0 pérdidas en los procesos y equipos dentro de un contexto laboral. Los accidentes laborales, en cambio, son aquellos hechos lesivos o mortales que tienen lugar durante la jornada de trabajo y que se caracterizan por ser violentos y repentinos, pero prevenibles 2 . Mientras que los accidentes son evitables, los riesgos están siempre presentes y a veces sólo es posible neutralizarlos o minimizar-los a través de capacitaciones y señalizaciones que cumplen una función preventiva más que anuladora. Por tanto, puede decirse que no hay puesto de trabajo que no conlleve riesgos laborales [10].

\section{Salud ocupacional}

La seguridad y salud ocupacional se preocupa por preservar y proteger los recursos humanos y de las instalaciones en el lugar de trabajo. La seguridad y salud en el trabajo implica ayudar a las personas evitando que se lesionen o se enfermen debido a los peligros en sus lugares de trabajo. La seguridad y salud ocupacional es también un campo en el que los profesionales intentan prevenir pérdidas catastróficas. En la práctica, la seguridad y salud en el trabajo incluye cuestiones morales y económicas [11].

Los requisitos de seguridad para el programa de salud y seguridad incluyen sistemas para el compromiso y las responsabilidades de la gerencia; evaluación y control de peligros y planificación de seguridad, reglas, procedimientos de trabajo y capacitación que estén en su lugar y que funcionen de manera efectiva.

\section{Metodología}

La presente investigación sigue un enfoque cuantitativo, es descriptiva y transversal.

El objetivo principal de la investigación es realizar un estudio diagnóstico sobre la salud organizacional de dos instituciones educativas, visto desde la perspectiva de sus trabajadores, a partir de la era covid-19 con el fin de saber en qué medida las organizaciones establecen acciones sobre gestión de salud laboral

El instrumento utilizado es una guía de autoevaluación para promover estrategias de empresa saludable desarrollada por [12], todo ello con el fin de lograr un 
primer acercamiento que nos permita obtener conclusiones preliminares sobre la apreciación que tiene la masa laboral dentro de la experiencia vivida durante la pandemia por Covid-19.

La población objeto de estudio estuvo compuesta por dos instituciones públicas de educación superior mexicanas, conformando una población total de 100 trabajadores (maestros, profesores investigadores y personal administrativo) quienes estuvieron interesados en participar. Se aplicó un muestreo por conveniencia, ya que por las circunstancias mismas de la pandemia por COVID19 fue difícil el acceso y contar con el tiempo disponible por parte de los encuestados, así mismo, se consideraron 50 casos por cada instituciones para que el análisis fuera equitativo y consistente. Es importante mencionar que el criterio de muestreo por conveniencia permite trabajar con aquellos casos que tengan la disponibilidad de colaborar con la investigación [13].

El instrumento de medición original está compuesto de 5 dimensiones: 1. Política y compromiso con la salud (23 reactivos) 2. Gestión de la prevención de riesgos laborales (22 reactivos) 3. Gestión de la promoción de la salud en el trabajo (14 reactivos) 4. Gestión de regreso al trabajo tras una baja por motivos de salud (10 reactivos) 5 . Política de bienestar laboral (22 reactivos), no obstante se seleccionaron únicamente 6 reactivos por cada dimensión con el propósito de únicamente considerar aspectos que están relacionados con las instituciones objeto de estudio y que pueden ser respondidas por los participantes, conformando entonces un total de 30 preguntas.

La utilización del cuestionario es relativamente sencilla, ya que en cada uno de los cinco apartados se proponen una serie de afirmaciones o ítems y se pide una respuesta en una escala de 0 a 3 para expresar hasta qué punto lo que se afirma es o no una práctica de gestión en la empresa, tal como se explica a continuación:

El valor 3 significa que es una práctica consolidada en la empresa, es decir, es un objetivo alcanzado totalmente.

El valor 2 significa que es una práctica en proceso de implantación, es decir, ya se han alcanzado progresos considerables.

El valor 1 significa que hay algunos intentos aislados de implantación, o sea que no se dan muchos progresos visibles.

El valor 0 significa que no se le presta atención en la práctica, es decir aún no forma parte de los objetivos de la empresa.

\section{Resultados}

En aras de no ser repetitivo y describir el significado de la nomenclatura utilizada en todos los cuadros, se tiene que:

Columna 1: Contiene la escala de puntuación de cada pregunta, la cual oscila de 0 a 3 y con lo cual calificaron cada pregunta los encuestados.

Columnas 2, 4, 6, 8, 10 y 12: Contiene el número total de encuestados que eligieron cada opción de respuesta en las 6 preguntas que componen la dimensión.

Columna 3, 5, 7, 9, 12 y 15: Total de puntuaciones obtenidas por cada pregunta al multiplicarse por el valor de cada opción elegida por los encuestados.

En el cuadro 2 se aprecia la puntuación obtenida en relación con la primera dimensión sobre política y compromiso con la salud, donde las 6 preguntas aplicadas están asociadas con la percepción que tienen los trabajadores sobre la existencia de una gestión integral de salud, la responsabilidad de los directivos al asumir los compromisos asociados, la existencia de procedimientos, la identificación de un responsable que coordina las diversas acciones, la efectividad de las estrategias planeadas en materia de salud y la evaluación recurrente.

El porcentaje total de cumplimiento de la primera dimensión se obtiene mediante la aplicación de la siguiente fórmula [12]:

Puntuación obtenida x 100\% / máxima puntuación posible.

$\frac{810 \times 100 \%}{1800}=45.0 \%$

Como se puede observar el porcentaje obtenido es menor al 50\%, lo que refleja una percepción media-baja por parte de los trabajadores en relación con la falta de compromiso por parte de los directivos para establecer estrategias de atención a la gestión integral de la salud, sobre todo durante la pandemia de Covid-19.

\begin{tabular}{|c|c|c|c|c|c|c|c|c|c|c|c|c|}
\hline Puntos & P-1 & $\begin{array}{c}\text { T-P- } \\
1 \\
\end{array}$ & P-2 & $\begin{array}{c}\text { T-P- } \\
2 \\
\end{array}$ & $\mathrm{P}-3$ & $\begin{array}{c}\text { T-P- } \\
3 \\
\end{array}$ & P-4 & $\begin{array}{c}\text { T-P- } \\
4\end{array}$ & P-5 & $\begin{array}{c}\text { T-P- } \\
5\end{array}$ & $P-6$ & $\begin{array}{c}\text { T-P- } \\
6 \\
\end{array}$ \\
\hline 3 & 25 & 75 & 31 & 93 & 20 & 60 & 18 & 54 & 22 & 66 & 17 & 51 \\
\hline 2 & 17 & 34 & 19 & 38 & 25 & 50 & 17 & 34 & 18 & 36 & 18 & 36 \\
\hline 1 & 26 & 26 & 19 & 19 & 30 & 30 & 42 & 42 & 33 & 33 & 33 & 33 \\
\hline 0 & 32 & 0 & 31 & 0 & 25 & 0 & 23 & 0 & 27 & 0 & 32 & 0 \\
\hline Total & 100 & 135 & $\begin{array}{c}10 \\
0\end{array}$ & 150 & $\begin{array}{c}10 \\
0\end{array}$ & 140 & $\begin{array}{c}10 \\
0\end{array}$ & 130 & $\begin{array}{c}10 \\
0\end{array}$ & 135 & $\begin{array}{c}10 \\
0\end{array}$ & 120 \\
\hline
\end{tabular}

Cuadro 2. Política y compromiso con la salud. 
Fuente: Elaboración propia con base a datos obtenidos.

En cuadro 3 se muestra la puntuación obtenida en relación con la segunda dimensión sobre Gestión de la prevención de riesgos laborales, donde las 6 preguntas aplicadas están asociadas con la percepción que tienen los trabajadores sobre la igualdad en importancia que se le da en la organización a la productividad y a la salud y seguridad, si los trabajadores participan en la toma de decisiones sobre el tema que nos ocupa, la consideración, evaluación y control que se le da a los riesgos laborales, la responsabilidad individual y promoción de la cultura en materia de salud en el trabajo.

El porcentaje total de cumplimiento de la segunda dimensión se obtiene mediante la aplicación de la siguiente fórmula:

Puntuación obtenida x 100\% / máxima puntuación posible.

El porcentaje total de cumplimiento de la segunda dimensión se obtiene mediante la aplicación de la siguiente fórmula:

Puntuación obtenida x 100\% / máxima puntuación posible.

$\frac{790 \times 100 \%}{1800}=43.9 \%$

Como se puede observar el porcentaje obtenido es menor al $50 \%$, lo que refleja una percepción media-baja por parte de los trabajadores en relación con la falta de una adecuada gestión por parte de los directivos para establecer estrategias de prevención de riesgos laborales que puedan impactar en la salud del personal, sobre todo durante la pandemia de Covid-19.

\begin{tabular}{|c|c|c|c|c|c|c|c|c|c|c|c|c|}
\hline untos & P-1 & $\begin{array}{c}\text { T-P. } \\
1\end{array}$ & $\mathrm{P}-2$ & $\begin{array}{c}\text { T-P. } \\
2\end{array}$ & $\mathrm{P}-3$ & $\begin{array}{c}\text { T-P. } \\
3\end{array}$ & P-4 & $\begin{array}{c}\text { T-P. } \\
4\end{array}$ & P-5 & $\begin{array}{c}\text { T.P. } \\
5\end{array}$ & P-6 & T-P. \\
\hline 3 & 17 & 51 & 16 & 48 & 22 & 66 & 21 & 63 & 29 & 87 & 25 & 75 \\
\hline 2 & 20 & 40 & 12 & 24 & 11 & 22 & 16 & 32 & 21 & 42 & 18 & 36 \\
\hline 1 & 39 & 39 & 38 & 38 & 32 & 32 & 30 & 30 & 36 & 36 & 29 & 29 \\
\hline 0 & ${ }^{2}$ & 0 & 34 & & 35 & & 33 & 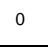 & 14 & 0 & 28 & 0 \\
\hline Total & 100 & 130 & $\begin{array}{l}10 \\
0\end{array}$ & 110 & 10 & 120 & $\begin{array}{c}10 \\
0\end{array}$ & 125 & $\begin{array}{l}10 \\
0\end{array}$ & 165 & $\begin{array}{c}10 \\
0\end{array}$ & 140 \\
\hline
\end{tabular}

Cuadro 3. Gestión de la prevención de riesgos laborales.

Fuente: Elaboración propia con base a datos obtenidos.

En el cuadro 4 se muestra la puntuación obtenida en relación con la tercera dimensión sobre Gestión de la promoción de la salud en el trabajo, donde las 6 preguntas aplicadas están asociadas con la percepción que tienen los trabajadores sobre la aplicación de programas específicos de salud sobre alimentación, actividad física, estrés, tabaco, entre otras, así como sus beneficios, si se pone a disposición los servicios y/o equipo especial para llevar a cabo las actividades anteriores, se promociona el buen clima laboral, control y mejora de los riesgos laborales, así como apoyo a iniciativas sociales a favor de la promoción de la salud laboral.

El porcentaje total de cumplimiento de la tercera dimensión se obtiene mediante la aplicación de la siguiente fórmula:

Puntuación obtenida x 100\% / máxima puntuación posible.

$\frac{785 \times 100 \%}{1800}=43.6 \%$

Como se puede observar el porcentaje obtenido es menor al $50 \%$, lo que refleja una percepción media-baja por parte de los trabajadores en relación con la falta de una adecuada gestión por parte de los directivos para establecer estrategias de promoción de de la salud en el trabajo, sobre todo durante la pandemia de Covid-19.

\begin{tabular}{|c|c|c|c|c|c|c|c|c|c|c|c|c|}
\hline Puntos & P-1 & $\begin{array}{c}\text { T-P- } \\
1\end{array}$ & $\mathrm{P}-2$ & $\begin{array}{c}\text { T-P- } \\
2\end{array}$ & P-3 & $\begin{array}{c}\text { T-P. } \\
3\end{array}$ & P-4 & $\begin{array}{c}\text { T-P. } \\
4\end{array}$ & P-5 & $\begin{array}{c}\text { T-P. } \\
5\end{array}$ & P-6 & $\begin{array}{c}\text { T-P. } \\
6\end{array}$ \\
\hline 3 & 20 & 60 & 22 & 66 & 19 & 57 & 26 & 78 & 20 & 60 & 22 & 66 \\
\hline 2 & 19 & 38 & 20 & 40 & 15 & 30 & 20 & 40 & 20 & 40 & 14 & 28 \\
\hline 1 & 27 & 27 & 29 & 29 & 33 & 33 & 17 & 17 & 30 & 30 & 46 & 46 \\
\hline 0 & 34 & 0 & 29 & 0 & 33 & & 37 & 0 & 30 & & 18 & 0 \\
\hline Total & 100 & 125 & $\begin{array}{c}10 \\
0\end{array}$ & 135 & 10 & 120 & 10 & 135 & $\begin{array}{c}10 \\
0\end{array}$ & 130 & $\begin{array}{c}10 \\
0\end{array}$ & 140 \\
\hline
\end{tabular}

Cuadro 4. Gestión de la promoción de la salud en el trabajo.

Fuente: Elaboración propia con base a datos obtenidos.

En el cuadro 5 se muestra la puntuación obtenida en relación con la cuarta dimensión sobre Gestión de regreso al trabajo tras una baja por motivos de salud, donde las 6 preguntas aplicadas están asociadas con la percepción que tienen los trabajadores en relación a la comunicación que existe con ellos cuando por algún 
motivo se encuentran de incapacidad médica, interés sobre la evolución de su salud, presión para regresar pronto a laborar, barreras y dificultades desde el seno familiar, apoyo y seguimiento.

El porcentaje total de cumplimiento de la cuarta dimensión se obtiene mediante la aplicación de la siguiente fórmula:

Puntuación obtenida x 100\% / máxima puntuación posible.

$\frac{720 \times 100 \%}{1800}=40.0 \%$

Como se puede observar el porcentaje obtenido es menor al $50 \%$, lo que refleja una percepción media-baja por parte de los trabajadores en relación con la falta de una adecuada gestión por parte de los directivos para establecer estrategias asociadas para el regreso al trabajo tras una baja por motivos de salud, sobre todo durante la pandemia de Covid-19.

\begin{tabular}{|c|c|c|c|c|c|c|c|c|c|c|c|c|}
\hline Intos & P-1 & $\begin{array}{c}\text { T.P. } \\
1\end{array}$ & P-2 & $\begin{array}{c}\text { T-P. } \\
2\end{array}$ & $\mathrm{P}-3$ & $\begin{array}{c}\text { T-P. } \\
3\end{array}$ & P-4 & $\begin{array}{c}\text { T.P. } \\
4\end{array}$ & P-5 & $\begin{array}{c}\text { T-P- } \\
5\end{array}$ & P-6 & $\begin{array}{c}\text { T-P. } \\
6\end{array}$ \\
\hline 3 & 15 & 45 & 14 & 42 & 13 & 39 & 20 & 16 & 21 & 63 & 16 & 48 \\
\hline 2 & 26 & 52 & $2 J$ & 50 & & 56 & 30 & 60 & 21 & 42 & 16 & 32 \\
\hline 1 & 23 & 23 & 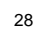 & 28 & 25 & 25 & 44 & 44 & 25 & 25 & 30 & 30 \\
\hline 0 & 36 & o & 33 & 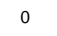 & 34 & 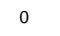 & 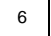 & 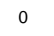 & 33 & 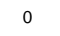 & 38 & 0 \\
\hline Total & 100 & 120 & $\begin{array}{c}10 \\
0\end{array}$ & 120 & 10 & 120 & $\begin{array}{c}10 \\
0\end{array}$ & 120 & $\begin{array}{c}10 \\
0\end{array}$ & 130 & $\begin{array}{c}10 \\
0\end{array}$ & 110 \\
\hline
\end{tabular}

Cuadro 5. Gestión de regreso al trabajo tras una baja por motivos de salud.

Fuente: Elaboración propia con base a datos obtenidos.

En el cuadro 6 se muestra la puntuación obtenida en relación con la quinta dimensión sobre Política de bienestar laboral, donde las 6 preguntas aplicadas están asociadas con la percepción que tienen los trabajadores en relación al trato justo y equitativo, manejo adecuado de conflictos, promoción del entusiasmo al cumplimiento de objetivos, si el personal entiende el sentido de sus tareas y tiene las mismas oportunidades de crecer y desarrollarse profesionalmente en el trabajo, así como el grado de compromiso con la mejora continua.

El porcentaje total de cumplimiento de la quinta dimensión se obtiene mediante la aplicación de la siguiente fórmula:

Puntuación obtenida x 100\% / máxima puntuación posible.

$\frac{840 \times 100 \%}{1800}=46.7 \%$

\begin{tabular}{|c|c|c|c|c|c|c|c|c|c|c|c|c|}
\hline Puntos & P-1 & $\begin{array}{c}\text { TT-P. } \\
1\end{array}$ & P-2 & $\begin{array}{c}\text { T-P. } \\
2\end{array}$ & P-3 & $\begin{array}{c}\text { TT-P. } \\
3\end{array}$ & P-4 & $\begin{array}{c}\text { T-P- } \\
4\end{array}$ & P-5 & $\begin{array}{c}\text { T-P- } \\
5\end{array}$ & P-6 & $\begin{array}{c}\text { T-P- } \\
6\end{array}$ \\
\hline 3 & 20 & 60 & 25 & 75 & 25 & 75 & 30 & 90 & 27 & 81 & 26 & 78 \\
\hline 2 & 20 & 40 & 17 & 34 & 10 & 20 & 10 & 20 & 18 & 36 & 13 & 26 \\
\hline 1 & 30 & 30 & 21 & 21 & 45 & 45 & 30 & 30 & 33 & 33 & 46 & 46 \\
\hline 0 & 30 & 0 & 37 & 0 & 20 & 0 & 30 & 0 & 22 & 0 & 15 & 0 \\
\hline Total & 100 & 130 & $\begin{array}{c}10 \\
0\end{array}$ & 130 & $\begin{array}{c}10 \\
0\end{array}$ & 140 & $\begin{array}{c}10 \\
0\end{array}$ & 140 & $\begin{array}{c}10 \\
0\end{array}$ & 150 & $\begin{array}{c}10 \\
0\end{array}$ & 150 \\
\hline
\end{tabular}

Cuadro 6. Política de bienestar laboral.

Fuente: Elaboración propia con base a datos obtenidos. Como se puede observar el porcentaje obtenido es menor al $50 \%$, lo que refleja una percepción media-baja por parte de los trabajadores en relación con la falta de una adecuada gestión por parte de los directivos para establecer Políticas de bienestar laboral, sobre todo durante la pandemia de Covid-19.

Finalmente, se puede aplicar la misma fórmula que se utilizó en cada dimensión sumando las puntuaciones obtenidas y dividiéndolas entre la máxima puntuación posible del instrumento en su conjunto, quedando de la siguiente manera:

Suma de todas las puntuaciones obtenidas de cada dimensión x 100\% / máxima puntuación posible de todo el instrumento.

$\frac{3945 \times 100 \%}{9000}=43.8 \%$ 
El resultado de $44.0 \%$ indica el nivel de salud de las instituciones educativas participantes, lo cual es un indicativo muy importante para la toma de decisiones de los directivos que deseen desarrollar un cultura de salud laboral, sobre todo en estos tiempos de pandemia provocada por el Covid-19.

\section{Conclusiones y discusión}

A pesar de que el resultado final obtenido (44\%) se ubica en un rango medio de $33-66 \%$, de acuerdo con [12], la atención debe centrarse en mejorar los planes para el desarrollo de actividades en todas las 5 dimensiones de la gestión de salud laboral, incorporando acciones efectivas de prevención, promoción e intervención con la participación activa de los trabajadores, alumnos y gente externa entusiasta que desee colaborar en algunas actividades sociales.

Las universidades deben hacer de la seguridad y la salud de su personal, su máxima prioridad. A partir del brote de COVID-19, se deben tomar medidas inmediatas para elaborar planes adecuados a su contexto en particular [14].

La promoción del bienestar laboral es una de las dimensiones quizás más importantes y en el estudio realizado se refleja con un nivel que se encuentra por debajo de la media, por lo que los directivos de las empresas educativas encuestadas deberán ayudar a proteger al personal y evitar la propagación de COVID19, además de ofrecer un nivel de bienestar positivo permanente [15].

Finalmente, es importante mencionar que existe poca información sobre investigaciones dirigidas a profesores universitarios en América Latina que aborden los factores de riesgo psicosocial ocupacional y sus efectos en el sector académico universitario, por lo que es necesario investigar más a fondo las interacciones de las condiciones laborales y las demandas sicológicas particulares a las que responden los profesores universitarios, ya que esto permitirá identificar el mecanismo de las enfermedades profesionales, sus costos e implicaciones para el cumplimiento de su misión educativa [16]

\section{Referencias}

[1] Nair, H., Kumar, D. y Ramalu, S. (2014). Organizational Health: Delineation, Constructs and Development of a Measurement Model. Asian Social Science, 10(14), 145-157. http://dx.doi.org/10.5539/ass.v10n14p145

[2] Şenol, L. y Ferhatoğlu, M. (2019). Occupational health and safety in businesses. Social Sciences Studies Journal, 5(50), 6691-6700. http://dx.doi.org/10.26449/sssj.1915

[3] Hamouche, S. (2020). COVID-19 and employees' mental health: stressors, moderators and agenda for organizational actions. Emerald Open Research, 2(15), 1-11. http://doi.org/10.35241/emeraldopenres.13550.1

[4] Cucinotta, D. y Vanelli, M. (2020). WHO Declares COVID-19 a Pandemic. Acta Biomédica, 91(1), 157-160. http://doi.org/10.23750/abm.v91i1.9397

[5] Benavides, F. G. (2020). La salud de los trabajadores y la COVID19. Archivos de prevención de riesgos laborales, 23(2), 154-158. https://scielo.isciii.es/scielo.php?script=sci_arttext\&pid=S157825492020000200002

[6] CEPAL, N. (2020, 21 de abril). Dimensionar los efectos del COVID-19 para pensar en la reactivación. Naciones Unidas. https://repositorio.cepal.org/bitstream/handle/11362/45445/S2000286_ es.pdf?sequence $=4 \&$ is Allowed $=y$

[7] Salanova, M. (2020). How to survive Covid-19? Notes from organisational resilience (¿Cómo sobrevivir al Covid-19? Apuntes desde la resiliencia organizacional). International Journal of Social Psychology, 35(3), 670-676. https://www.tandfonline.com/doi/full/10.1080/02134748.2020.179539

[8] Valenzuela, L.M. (2016). La salud, desde una perspectiva integral. Revista Universitaria de la Educación Física y el Deporte, 9(9), 30-59. file://C:/Users/CAJA-2/AppData/Local/Temp/DialnetLaSaludDesdeUnaPerspectivaIntegral-6070681.pdf

[9] Corbett, D. (2004). Excellence in Canada: Healthy organisations Achieve results by acting responsibly. Journal of Business Ethics, 55(1): 125-133. http://doi.org/10.1007/s10551-004-1896-8

[10] Revisión histórica de la salud ocupacional y la seguridad industrial. Revista Cubana de Salud y Trabajo, 13(3), 45-52. https://www.medigraphic.com/pdfs/revcubsaltra/cst-2012/cst123g.pdf

[11] Ayalew, A. y Demissie, Y. (2020). The Effect Of Occupational Health And Safety Program On Organizational Productivity: In Case Of Bahirdar Tannery Factory. International Journal of Scientific and $\begin{array}{lll}\text { Research } & \text { Publications, } & \text { 70(2), }\end{array}$ http://dx.doi.org/10.29322/IJSRP.10.02.2020.p98100

[12] IBERMUTUA (2020). Guía de autoevaluación para promover estrategias de empresa saludable. https://prevencionriesgoslaboralescev.es/wp- 
content/uploads/2019/11/Gu\%C3\%ADa-de-autoevaluaci\%C3\%B3npara-promover-estrategias-de-empresa-saludable.pdf

[13] Hernández, R., Fernández, C. y Baptista, M. (2010). Metodología de la Investigación. McGraw Hill.

[14] Young, Q. (2020, 4 de abril). Time for universities to show their commitment to society. University World News. https://www.universityworldnews.com/post.php?story=202004011548 15248

[15] Benzian, H., Beltrán-Aguilar, E. y Niederman, R. (2020). Systemic Management of Pandemic Risks in Dental Practice: A Consolidated Framework for COVID-19 Control in Dentistry. Frontioers in Medicine, 8(1), 1-7. https://doi.org/10.3389/fmed.2021.644515

[16] Monroy, A.y Juárez, A. (2019). Occupational Psychosocial Risk Factors in Academics of Higher Education Institutions in Latin America: a Systematic Review. Revista Propósitos y Represnetaciones, 7(3), 248-272. http://dx.doi.org/10.20511/pyr2019.v7n3.361 